University of Wollongong

Research Online

Faculty of Engineering - Papers (Archive)

Faculty of Engineering and Information

Sciences

$1-1-2012$

\title{
Study of vacancy-type defects by positron annihilation in ultrafine-grained aluminum severely deformed at room and cryogenic temperatures
}

LH. Su

University of Wollongong, lihongsu@uow.edu.au

Cheng Lu

University of Wollongong, chenglu@uow.edu.au

Lizi He

Northeastern University

L C. Zhang

University Of Western Australia

P Guagliardo

University of Western Australia

See next page for additional authors

Follow this and additional works at: https://ro.uow.edu.au/engpapers

Part of the Engineering Commons

https://ro.uow.edu.au/engpapers/5091

\section{Recommended Citation}

Su, L H.; Lu, Cheng; He, Lizi; Zhang, L C.; Guagliardo, P; Tieu, A K.; Samarin, S N.; Williams, J F.; and Li, H J.: Study of vacancy-type defects by positron annihilation in ultrafine-grained aluminum severely deformed at room and cryogenic temperatures 2012, 4218-4228.

https://ro.uow.edu.au/engpapers/5091

Research Online is the open access institutional repository for the University of Wollongong. For further information contact the UOW Library: research-pubs@uow.edu.au 


\section{Authors}

L H. Su, Cheng Lu, Lizi He, L C. Zhang, P Guagliardo, A K. Tieu, S N. Samarin, J F. Williams, and H J. Li 


\title{
Study of vacancy-type defects by positron annihilation in ultrafine-grained aluminum severely deformed at room and cryogenic temperatures
}

\author{
L.H. Su ${ }^{\mathrm{a}, \mathrm{b}}$, C. Lu ${ }^{\mathrm{a}, *}$, L.Z. He ${ }^{\mathrm{b}}$, L.C. Zhang ${ }^{\mathrm{c}}$, P. Guagliardo ${ }^{\text {d }}$, A.K. Tieu ${ }^{\mathrm{a}}$, \\ S.N. Samarin ${ }^{\text {d, J.F. Williams }}{ }^{\text {d }}$, H.J. Li ${ }^{\text {a }}$ \\ ${ }^{a}$ School of Mechanical, Materials and Mechatronic Engineering, University of Wollongong, Wollongong, NSW 2522, Australia \\ ${ }^{\mathrm{b}}$ School of Materials and Metallurgy, Northeastern University, Shenyang 110004, People's Republic of China \\ ${ }^{\mathrm{c}}$ School of Mechanical Engineering, The University of Western Australia, 35 Stirling Highway, Crawley, WA 6009, Australia \\ d ARC Centre of Excellence for Antimatter-Matter Studies, School of Physics, The University of Western Australia, \\ 35 Stirling Highway, Crawley, WA 6009, Australia
}

Received 20 December 2011; received in revised form 25 March 2012; accepted 1 April 2012

Available online 18 May 2012

\begin{abstract}
Commercial-purity aluminum was processed by equal-channel angular pressing (ECAP) at room temperature (RT-ECAP) and cryogenic temperature (CT-ECAP) with liquid nitrogen cooling between two successive passes. It was found that the RT-ECAPed samples showed equiaxed microstructure after 4 and 8 ECAP passes, while the CT-ECAPed samples displayed slightly elongated microstructure and slightly smaller grain size. Moreover, the CT-ECAPed samples had higher hardness values than the RT-ECAPed samples subjected to the same amount of deformation. Positron annihilation lifetime spectroscopy (PALS) was used to investigate the evolution of vacancytype defects during the ECAP deformation process. The results showed that three types of defects existed in the ECAPed samples: vacancies associated with dislocations, bulk monovacancies and bulk divacancies. The CT-ECAPed samples had a higher fraction of monovacancies and divacancies. These two types of defects are the major vacancy-type defects that can work as dislocation pinning centers and induce hardening, resulting in higher hardness values in the CT-ECAPed samples. A quantitative relationship between material hardness and the defect concentration and defect diffusion coefficient has been established.
\end{abstract}

(C) 2012 Acta Materialia Inc. Published by Elsevier Ltd. All rights reserved.

Keywords: Equal-channel angular pressing (ECAP); Low-temperature deformation; Vacancies; Positron annihilation (PAL); Hardness

\section{Introduction}

Ultrafine-grained (UFG) materials, processed by severe plastic deformation (SPD), have attracted a great deal of attention in the last decade due to their superior mechanical properties, such as significantly enhanced strength and hardness. Special interest has been paid to the mechanism responsible for hardening in UFG materials. Two new work-hardening stages (Stages IV and V) have been

\footnotetext{
* Corresponding author.

E-mail address: chenglu@uow.edu.au (C. Lu).
}

identified in UFG materials and added to the other three stages (Stages I, II and III) often observed in their coarse-grained counterparts [1,2]. Dislocations, grain boundaries and their interactions dominate the work-hardening mechanisms in Stages I-IV, while the interaction of vacancy-type defects and dislocations has been regarded as the major work-hardening mechanism in Stage V [2]. In addition, our recent study [3] showed that vacancy-type defects are responsible for unusual phenomena such as annealing-induced hardening and softening by slight deformation in severely deformed Al. Thus, there is significant evidence that vacancy-type defects play an important role 
during the deformation of UFG materials. However, further investigations are required to elucidate the precise role of such defects in the deformation process.

Positron annihilation lifetime spectroscopy (PALS) is a powerful tool for studying vacancy-type defects in metals and alloys $[4,5]$. In this method, positrons from the decay of ${ }^{22} \mathrm{Na}$ are injected into a sample and a lifetime spectrum is recorded. Once implanted, the positron will come to thermal equilibrium in the material and will eventually annihilate with an electron, producing $\gamma$-quanta. Annihilation can occur in a number of different states, e.g. as a trapped positron localized in a vacancy-type defect (such as an atomic vacancy), or in the interstitial regions of the solid, characterized by a delocalized Bloch state. The lifetime of a positron trapped in an vacancy-type defect is enhanced relative to the bulk, owing to the reduced electron density at the defect site; this provides the basis for defect spectroscopy, and in principal both the type and the concentration of defects can be resolved.

Recently, PALS has been used to investigate vacancytype defects in UFG materials processed by two major SPD processes: equal-channel angular pressing (ECAP) and high-pressure torsion (HPT). Cížek et al. have studied UFG structures in $\mathrm{Cu}, \mathrm{Fe}, \mathrm{Ni}$ and $\mathrm{Mg}$ metals and alloys by means of PALS in combination with transmission electron microscopy (TEM), X-ray diffraction (XRD) and microhardness measurements. Defects were identified and their concentrations and spatial distributions were investigated. Vacancies situated in distorted regions along grain boundaries and small vacancy clusters distributed homogeneously inside dislocation-free grain interiors were observed [6-10]. Divinski et al. also conducted PALS on UFG Cu-1 wt.\% $\mathrm{Pb}$ alloy, which revealed the existence of vacancy-type defects, presumably related to grain boundary states, and vacancy clusters in this material [11]. Al alloys with UFG structure are the most important lightweight structural materials; however, they have been rarely studied by PALS except for Ref. [12], in which the nature of vacancy-type defects induced by deformation and vacancy-solute interactions in $\mathrm{Al}-\mathrm{Cu}-\mathrm{Mg}-\mathrm{Mn}$ alloys deformed by HPT was investigated.

The formation and migration of vacancy-type defects are strongly temperature-dependent. It is expected that low-temperature deformation can generate more vacancytype defects and in turn enhance the interactions between dislocations and vacancy-type defects and change the material properties [13]. A number of researches have shown that room temperature (RT) ECAP followed by cryogenic temperature $(\mathrm{CT})$ rolling increased both the strength and ductility of the materials [14-16]. Also, it has been demonstrated that CT ECAP processing alone can lead to the enhanced hardness compared with RT ECAP processing [17]. However, no investigation of the role of vacancy-type defects in UFG materials subjected to cryogenic SPD processes has been conducted.

In this study, commercial-purity $\mathrm{Al}$ was deformed by ECAP at both room temperature and cryogenic temperature. TEM observation and hardness tests were conducted to examine the microstructure and mechanical property evolution of the materials. In order to investigate the effect of vacancy-type defects on hardening in UFG Al alloys, a PALS investigation of the ECAPed samples was conducted. A detailed analysis was performed and a quantitative relationship between the material hardness and the defect concentration and defect diffusion coefficient has been established.

\section{Experiments}

The material used was commercially pure Al (AA1050). It was cast and heat treated at $456{ }^{\circ} \mathrm{C}$ for $1 \mathrm{~h}$ and then cut into $20 \times 20 \times 100 \mathrm{~mm}^{3}$ billets. The billets were pressed through an ECAP die with a pressing speed of $7 \mathrm{~mm} \mathrm{~s}^{-1}$. The intersection angle of the die is $90^{\circ}$ and the outer arc of curvature is $20^{\circ}$, providing an equivalent strain of approximately 1 per ECAP pass. The billets were deformed by up to 8 passes via route $B_{C}$. One group of the billets (RT-ECAPed samples) stayed at ambient atmosphere between two successive passes, while the second group of the billets (CT-ECAPed samples) were immersed in liquid nitrogen immediately after each pass for $1 \mathrm{~h}$ before the next pass. The die remained at ambient temperature during the ECAP deformation. After the final ECAP deformation the second group of the billets were left at ambient atmosphere to allow the temperature to reach room temperature.

The samples subjected to 1, 4 and 8 ECAP passes were selected for further testing. They are named RT-1, RT-4 and RT- 8 for the RT-ECAPed samples, and CT-1, CT-4 and CT-8 for the CT-ECAPed samples, respectively. Vickers microhardness was measured with a Leco hardness testing machine by applying a load of $100 \mathrm{~g}$ for $12 \mathrm{~s}$. TEM was used to observe the microstructural evolution of the ECAPed samples. Thin foils were first sliced from the plane perpendicular to the pressing direction then mechanically ground to a thickness of $<200 \mu \mathrm{m}$ and twin-jet electropolished with Struers Tenupol polishing machine in a solution of $20 \%$ nitric acid and $80 \%$ methanol at $-20{ }^{\circ} \mathrm{C}$. TEM observation was conducted on a JEOL 2011 microscope operated at $200 \mathrm{kV}$.

PALS measurements were carried out using a fast-fast spectrometer, with detectors consisting of a BC418 plastic scintillator coupled to a Burle 8850 photomultiplier tube. The positron source consisted of $\sim 30 \mu \mathrm{Ci}$ of ${ }^{22} \mathrm{NaCl}$ sealed in $7 \mu \mathrm{m}$ thick Kapton foil (grade HN from Goodfellow), and was sandwiched between two identical samples. The time resolution of the system is about $220 \mathrm{ps}$, as determined from analysis of a spectrum of high-purity annealed nickel. The spectra comprise of at least 2 million counts. An Al sample annealed at $600{ }^{\circ} \mathrm{C}$ for $1 \mathrm{~h}$ was measured to determine the source lifetime and intensity. Shukla et al. [18] have developed a program MELT 4.0 to analyze the positron lifetime. This program uses the maximum entropy method to extract positron lifetime distribution without any assumption as to the number of lifetime components. 
It has been proven that MELT 4.0 is especially appropriate for evaluating short positron lifetimes [19]. In this study, the MELT 4.0 program has been used to analyze the measured spectra.

\section{Results}

The results of hardness measurements on the RT- and CT-ECAPed samples are shown in Fig. 1. There is a significant increase in hardness after 1 ECAP pass at both temperatures. The hardness of the RT-ECAPed samples increases monotonically with the number of passes although the rate of the increment is reduced. For the CT-ECAPed samples, the hardness increases from sample CT-1 to sample CT-4 and then slightly decreases when the deformation proceeds to the 8 th pass. A comparison of the two curves in Fig. 1 clearly depicts that the samples subjected to cryogenic processing are harder than those deformed at room temperature.

TEM images of the ECAPed samples are shown in Fig. 2. It can be seen from Fig. 2a and $d$ that the microstructural features in samples RT-1 and CT-1 are similar; thus no immediate differences can be distinguished by TEM at this stage. Both samples consist of a network of dislocation cells with a high dislocation density surrounded by low-angle boundaries. The mean linear size of dislocation cells in samples is of the order of approximately $600 \mathrm{~nm}$. Fig. $2 \mathrm{~b}$ and e shows the microstructures of samples RT-4 and CT-4, respectively. It can be seen that the dislocation density is quite low in most grain interiors of the sample RT-4. Also, the grains in RT-4 appear to be fairly equiaxed and are surrounded by relatively high-angle grain boundaries. However, the microstructure of the sample CT-4 is less equiaxed and a relatively high dislocation density in some grain interiors can be seen. This indicates that the sample CT-4 is less evolved than the sample RT-4. Although the grain size of the sample CT-4 is slightly smaller, both samples possess a similar average grain size, which

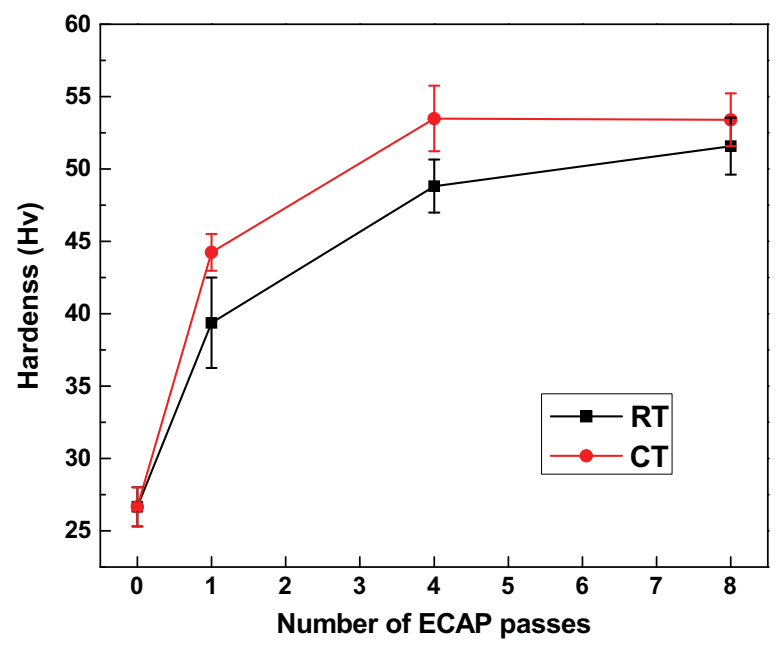

Fig. 1. Hardness measurements of ECAP-processed samples. is almost equal to the mean linear size of dislocation cells in samples RT-1 and CT-1. This suggests that the dislocation cells have developed into grains or subgrains. Grains in the sample RT-8 are reasonably equiaxed as shown in Fig. 2c and most of the grains are free of dislocations in the interiors and surrounded by high-angle grain boundaries. The microstructure of the sample CT-8, however, shows a less equiaxed grain shape. The average grain sizes of samples RT-8 and CT-8 are similar, which reveals that no obvious difference can be drawn from TEM observation at this magnitude. Although deformation at cryogenic temperature is expected to suppress dynamic recovery and further refine the microstructure, ECAP deformation of commercial-purity $\mathrm{Al}$ did not achieve this.

Positron lifetime analysis of the annealed Al sample reveals three lifetime components: 162,357 and 1770 ps. The first component (162 ps) locates within the range reported for the perfect lattice of aluminum [5,20]. The second and third components can be attributed to positron source annihilations. Staab et al. [21] stated that there are three different source contributions to the positron lifetimes. The first one is due to the fraction of the positrons annihilating in the support foil. McGuire and Keeble have studied the positron lifetime in Kapton support foil [22], and found a value of $\sim 369$ ps for this. The second source contribution is due to the radioactive salt and the interface between the foil and the sample. This contribution provides a lifetime of $\sim 350$ ps from analysis of the experimentally measured spectra [21]. The third source contribution, which generates a lifetime of about $1500-2500 \mathrm{ps}$, is due to the annihilation of positrons at surfaces [21]. The value of the third source lifetime depends on the size of the samples in comparison to the source size and the surface roughness of the samples. The second measured lifetime component ( $357 \mathrm{ps})$ in the annealed sample locates between the first (369 ps) and second ( $350 \mathrm{ps})$ source lifetimes. Since the two source lifetimes are too close to be separated in practice, it is reasonable to conclude that the second measured lifetime component is induced by the mixture of the first and second types of the source contributions. It is also clear that the measured longest lifetime of $1770 \mathrm{ps}$ is the third type of the source lifetime. The intensities for the second and third measured lifetimes are $15.0 \%$ and $0.2 \%$, respectively. They are close to those obtained from the experimental results and Monte Carlo simulations in Ref. [21]. The measured positron spectra of all ECAPed samples have been corrected by subtracting the source components (second and third lifetimes of the annealed sample).

Two positron lifetime components were obtained for the ECAPed samples: short lifetime $\tau_{1}$ and long lifetime $\tau_{2}$. These results are shown in Fig. $3 a$ and $b$, respectively. The error bars in the figures indicate the values of the full width at half maximum (FWHM) calculated by MELT. All of the $\tau_{1}$ values are smaller than the bulk Al lifetime ( $\sim 162 \mathrm{ps}$ ), while the $\tau_{2}$ values vary between 221 and 251 ps. It is known that vacancies can form by collisions 

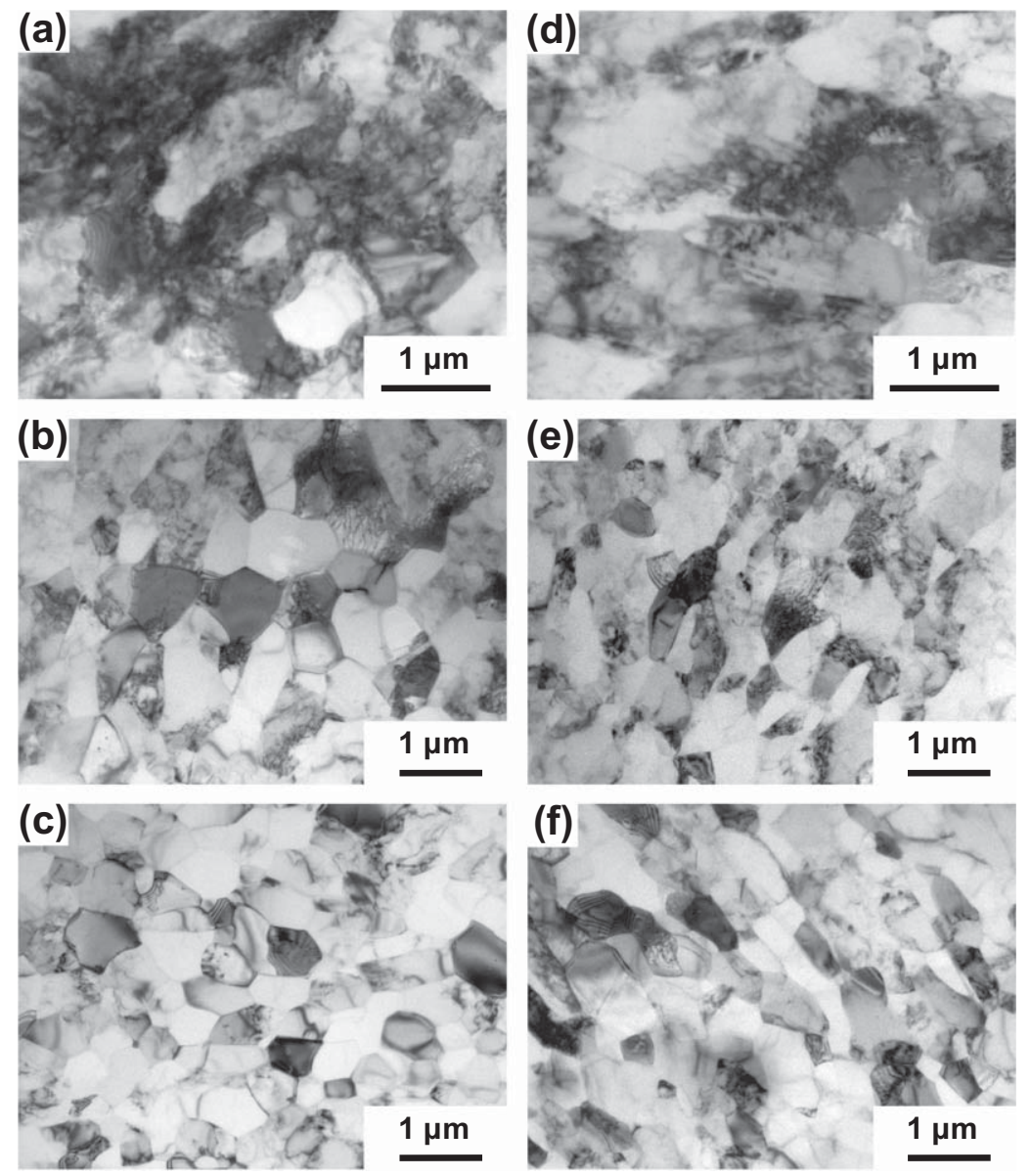

Fig. 2. TEM observations of ECAPed samples: (a) RT-1, (b) RT-4, (c) RT-8, (d) CT-1, (e) CT-4 and (f) CT-8.

of moving dislocations and they can easily diffuse into the dislocation cores. The positron lifetime of vacancies in the dislocation cores, which are also called vacancies associated with dislocations, is different from that of monovacancies in the bulk [23-25]. Petersen et al. [23] have reported a lifetime of $\sim 220 \mathrm{ps}$ in Al single crystal subjected to uniaxial tensile strain of up to $28 \%$, where dislocations were practically the sole type of defect produced. Therefore, $220 \mathrm{ps}$ is regarded as the positron lifetime in vacancies associated with dislocations. A similar result has been obtained by Hidalgo et al. [20], where two Al single crystal samples with different crystallographic orientations were strained $27 \%$ under tension. The positron lifetimes were found to be in the range of $215-220$ ps. In addition, Rajainmäkit and Linderoth [24] measured a lifetime spectrum for Al single crystal at $327^{\circ} \mathrm{C}$. At this temperature it is known that thermal monovacancies are present, and the lifetime of bulk monovacancies was found to be 246 ps. Fluss et al. [25] reported a similar lifetime of 244 ps for bulk monovacancies in high-purity polycrystalline measured at temperatures higher than $350^{\circ} \mathrm{C}$. Furthermore, Häkkinen et al. [26] studied the positron annihilation characteristics of vacancies in Al using molecular dynamics. They found that vacancy-type defects associated with $\left[\begin{array}{lll}1 & 1 & 2\end{array}\right]$ dislocations have positron lifetimes of $224-225$ ps, while the positron lifetime in a bulk monovacancy is $252 \mathrm{ps}$. Therefore, it has been generally accepted that vacancies associated with dislocations have a lifetime of $\sim 220$ ps, while bulk monovacancies have a lifetime of $\sim 245$ ps in Al.

Fig. 3a shows that all ECAPed samples possess a $\tau_{2}$ value of 220-245 ps, except for the sample CT-4 with $\tau_{2}=251 \mathrm{ps}$. It should be noted that it is difficult, if not impossible, for the lifetime analysis program to distinguish these two lifetimes given the very small difference between them. It is reasonable to conclude that the measured long lifetime values for all ECAPed samples, except for the sample CT-4, are the average lifetimes mainly due to positron annihilation in vacancies associated with dislocations and bulk monovacancies. Therefore, the fractions of these two vacancy-type defects can be estimated by $f_{1} \times 220+$ $f_{2} \times 245=\tau_{2}$ and $f_{1}+f_{2}=1$, where $f_{1}$ is the fraction of vacancies associated with dislocations and $f_{2}$ is the fraction of bulk monovacancies. The calculated fractions are listed in Table 1. Sample RT-1 has a $\tau_{2}$ value of $221 \mathrm{ps,} \mathrm{close} \mathrm{to}$ the lifetime for vacancies associated with dislocations (220 ps). The calculated $f_{1}$ value for this sample is $96 \%$, indicating that vacancies associated with dislocations are dominant. Samples RT-4, RT-8, CT- 1 and CT-8 have $\tau_{2}$ values between 220 and 245 ps. The $f_{2}$ values for these samples exceed $70 \%$, indicating that bulk monovacancies are 

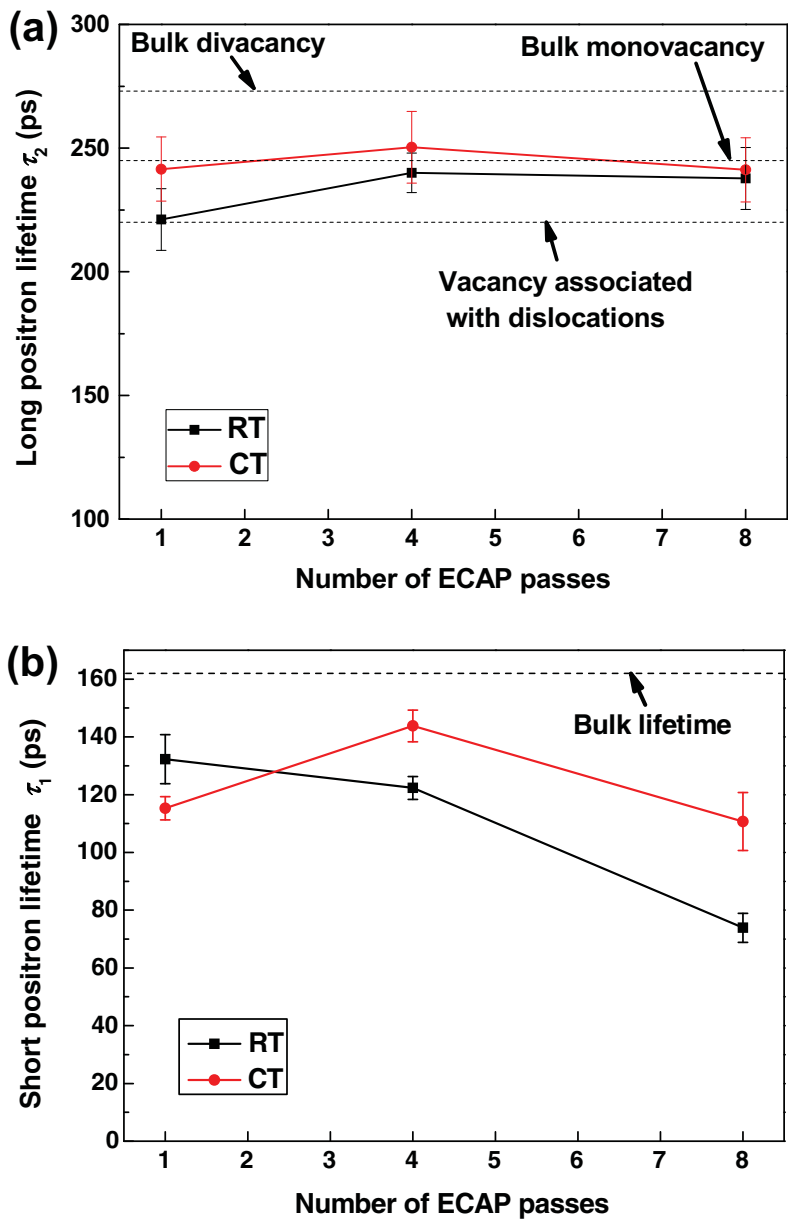

Fig. 3. Measured positron lifetimes of the ECAPed samples: (a) long lifetime and (b) short lifetime.

Table 1

Fractions of vacancy-type defects.

\begin{tabular}{|c|c|c|c|}
\hline \multirow[t]{2}{*}{ Sample } & \multicolumn{3}{|l|}{ Fraction (\%) } \\
\hline & $\begin{array}{l}\text { Vacancies associated } \\
\text { with dislocations }\left(f_{1}\right)\end{array}$ & $\begin{array}{l}\text { Monovacancies in } \\
\text { the bulk }\left(f_{2}\right)\end{array}$ & $\begin{array}{l}\text { Divacancies in } \\
\text { the bulk }\left(f_{3}\right)\end{array}$ \\
\hline RT-1 & 95.6 & 4.4 & - \\
\hline RT-4 & 20.0 & 80.0 & - \\
\hline RT-8 & 29.2 & 70.8 & - \\
\hline CT-1 & 14.0 & 86.0 & - \\
\hline CT-4 & - & 81.1 & 18.9 \\
\hline CT-8 & $15.2(22.86)^{\mathrm{a}}$ & $84.8(70.30)$ & $-(6.84)$ \\
\hline
\end{tabular}

${ }^{\mathrm{a}}$ The values in parentheses are the results when divacancies are assumed to exist in the sample CT-8.

the major vacancy-type defects in these samples and a certain amount of vacancies associated with dislocations still exist. Sample CT-4 exhibits a larger $\tau_{2}$ value of $251 \mathrm{ps}$, greater than that for bulk monovacancy. It is known that the positron lifetime increases as the size of vacancy-type defects increases. Thus, $\tau_{2}=251 \mathrm{ps}$ indicates that vacancy clusters exist in the sample. The positron lifetime of a divacancy is $273 \mathrm{ps}$ for $\mathrm{Al}$ [27]. Therefore, it is reasonable to assume that both bulk monovacancies

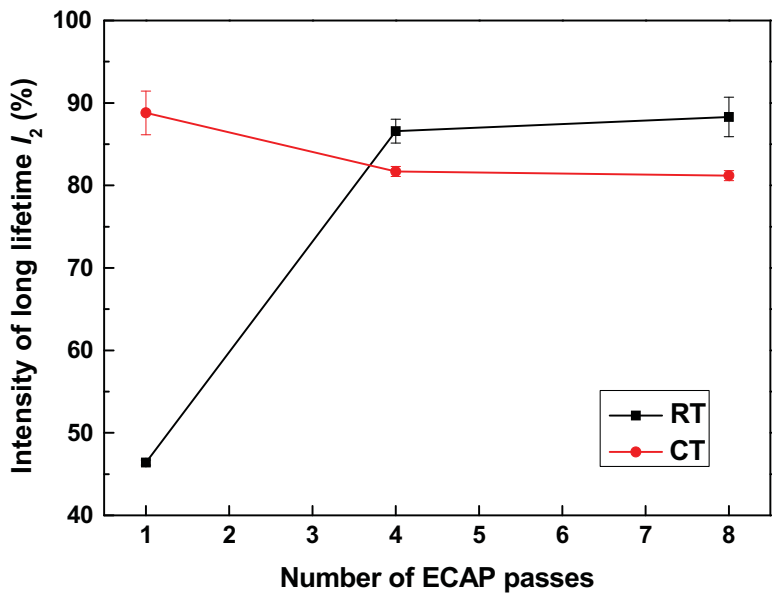

Fig. 4. Intensity of long positron lifetime of the ECAPed samples.

and bulk divacancies exist in the sample CT-4 and the fraction of vacancies associated with dislocations is relatively small. By assuming that $f_{1}$ is negligible the fractions of bulk monovacancies and divacancies are calculated as $81.1 \%$ and $19.9 \%$, respectively, determined by solving the equations $f_{2} \times 245+f_{3} \times 273=\tau_{2}$ and $f_{2}+f_{3}=1$ with $f_{3}$ being the fraction of bulk divacancies.

Fig. 3b shows the short positron lifetime $\left(\tau_{1}\right)$ for the ECAPed samples. The short positron lifetime can give a rough indication of the concentration of vacancy-type defects. A small $\tau_{1}$ value indicates a high defect concentration [9]. It can be seen that $\tau_{1}$ decreases monotonically with the number of ECAP passes for the RT-ECAPed samples, while it increases from sample CT- 1 to CT- 4 and then decreases from CT-4 to CT-8. Fig. 4 depicts the intensities $\left(I_{2}\right)$ of the long positron lifetime. $I_{2}$ significantly increases from sample RT-1 to RT-4 and then slightly increases for RT-8. For the CT-ECAPed samples, CT-1 has the largest $I_{2}$ value, followed by decreased $I_{2}$ in samples CT-4 and CT-8. $I_{2}$ is related to the concentrations and distributions of the vacancy-type defects. A detailed analysis is presented in the next section.

\section{Discussions}

Two mechanisms governing point defect formation during plastic deformation have been proposed $[28,13]$. First, if a positive dislocation and a negative dislocation come together and terminate one plane apart, a string of vacancies will be generated. If moving positive and negative dislocations terminate at the same plane, a string of self-interstitials will be generated. Second, if two moving dislocations cross, jogs on dislocations can be produced. Since the jog is subjected to the frictional drag under deformation, the jog velocity is lower than the velocity of an unjogged dislocation segment. Under these conditions nonconservative jog motion should result in the attendant production of point defects (vacancies or self-interstitials). The formation energy for a self-interstitial $(2.59 \mathrm{eV})$ is approximately four 
times that for a vacancy $(0.68 \mathrm{eV})$ [29], which suggests that it is hard to form self-interstitials in Al. Even if self-interstitials can be generated, they will easily annihilate in the sinks because the activation energy for migration of a self-interstitial $(0.1 \mathrm{eV}$ [28]) is much smaller than that for a vacancy $(0.61-0.64 \mathrm{eV}[29,30])$. Therefore, it is expected that the concentration of self-interstitials is negligible and vacancy-type defects are the common point defects in Al subjected to plastic deformation. During ECAP deformation the dislocations move and meet other dislocations, and act as both vacancy sources and vacancy sinks. When a dislocation encounters a bulk monovacancy, the monovacancy becomes a vacancy associated with the dislocation and moves along the dislocation core by pipe diffusion. The vacancy associated with the dislocation then annihilates when it reaches a vacancy sink, such as jog or grain boundary.

The fractions of vacancies associated with dislocations and bulk monovacancies are related to the dislocation density, the concentration of bulk monovacancies and the diffusion coefficient $(D)$ of bulk monovacancies. A higher dislocation density or a higher concentration of bulk monovacancies means that bulk monovacancies need to traverse a shorter distance before encountering a dislocation, thus resulting in a higher $f_{1}$ and a lower $f_{2}$. A higher $D$ indicates that a greater fraction of bulk monovacancies can readily migrate to the vicinity of the dislocation core and incorporate into the dislocation structure, also resulting in a higher $f_{1}$ value. $D$ can be described by the following equation, $D=D_{0} e^{-E_{M} / k T}$ [28], where $D_{0}$ is a constant, $E_{M}$ is the activation energy for the migration of a bulk monovacancy, $k$ is the Boltzmann constant and $T$ is the absolute temperature. It is clear that the vacancy diffusion coefficient increases with temperature. Thus, when the samples are deformed at room temperature, $D$ is high. The TEM image in Fig. 2a shows that the sample RT-1 is characterized by high dislocation density. Both a high vacancy diffusion coefficient and a high dislocation density give rise to a larger fraction of vacancies associated with dislocations in RT-1, as shown in Table 1. However, when the deformation is carried out at cryogenic temperature (sample CT1), the vacancy diffusion coefficient is low. Even though the sample CT-1 is also characterized by a high dislocation density, the low vacancy diffusion coefficient results in a lower fraction of vacancies becoming associated with dislocations. After 1 pass at cryogenic temperatures most monovacancies retain their identity as localized vacancy-type defects in the bulk rather than vacancies associated with dislocations. A fraction of bulk monovacancies may be annealed out during the subsequent temperature rise to room temperature. However, the temperature range for annealing of vacancy-type defects after low temperature deformation is appreciably higher than that after other vacancy-producing processes, such as irradiation [13]. The consequence is that the supersaturated bulk monovacancies remain in the sample CT-1 as reported in Table 1. Birnbaum [13] has also found that the vacancies in the sample deformed at $77 \mathrm{~K}$ do not become incorporated into the dislocation structure.

After 4 ECAP passes at room temperature, bulk monovacancies, rather than vacancies associated with dislocations, become the dominant vacancy-type defects as shown in Table 1. The reason can be attributed to the low dislocation density in the sample. The TEM observation in Fig. 2b shows that the sample RT-4 is characterized by relatively high-angle grain boundaries and a low dislocation density in the grain interiors. This suggests that compared to the sample RT-1, bulk monovacancies in RT-4 have less opportunity to meet dislocations, resulting in a higher fraction of bulk vacancies. For cryogenic ECAP processing, the dislocation density in the grain interiors decreases from the 1 st pass to the 4 th pass. However, since a high fraction of bulk monovacancies were generated in the first cryogenic ECAP pass, the concentration of bulk monovacancies increases continuously due to the accumulation of the dislocation-dislocation interactions. When the concentration of bulk monovacancies is high enough, the monovacancies should combine to form divacancies since the binding energy of two vacancies is positive [13]. Therefore, divacancies are likely to be the second major type of vacancy-type defects in the sample CT-4. Divacancies in Al are more mobile than monovacancies because the activation energy for migration is lower $(0.42-0.5 \mathrm{eV}$ for divacancies [30] compared to $0.61-0.64 \mathrm{eV}[30,31]$ for monovacancies). This indicates that some divacancies may annihilate in the grain boundaries.

Both the sample RT- 8 and CT- 8 are characterized by high-angle grain boundaries and a low dislocation density in the grain interiors. It has been found that the dislocation density in the 8 pass ECAPed samples is lower than that in the 4 pass ECAPed samples [31]. However, $f_{1}$ increases and $f_{2}$ decreases in RT-8 compared to RT-4. This indicates that the sample RT- 8 has a high concentration of bulk monovacancies. The low $\tau_{1}$ value for RT-8 shown in Fig. 3b confirms this conclusion. Details of the analysis will be given in the following text. Since the long positron lifetime of the sample CT-8 is located between that of bulk monovacancies and vacancies associated with dislocations, $f_{1}$ and $f_{2}$ for CT- 8 have been determined to be $15.2 \%$ and $84.8 \%$, respectively, from the measured long positron lifetime, assuming that the fraction of bulk divacancies is negligible. An intermediate level of divacancies was identified in the sample CT-4. It is difficult to conclude that divacancies disappear completely in the sample CT-8 even though they are more mobile than bulk monovacancies. However, the fraction of divacancies $\left(f_{3}\right)$ could not be determined from the sole long positron lifetime value.

The standard trapping model (STM) has been widely used to determine defect concentrations from positron lifetime data for metals and semiconductors [32]. This model assumes that defects are distributed homogeneously in the material. However, TEM observations in Fig. 2 show 
that dislocations are distributed inhomogeneously in both RT- and CT-ECAPed samples. A high dislocation density can be seen in the vicinity of the boundaries of dislocation cells and grains, while their interiors exhibit a relatively low dislocation density. This indicates that the STM cannot be directly applied to these samples. Dupasquier et al. [33] have developed a diffusion trapping model (DTM), which considers the positron diffusion from grain interiors to grain boundaries. This model assumes that the material is formed by identical spherical domains of radius $R$ and that open-volume defects only concentrate in a distorted region (DR) of constant thickness $(\delta)$ at the surfaces of the domains. This model was used for the analysis of positron lifetime spectra of a fine-grained $\mathrm{Al}-\mathrm{Ca}-\mathrm{Zn}$ alloy [33]. However, the assumption of defect-free grain interiors (non-distorted region (NR)) is inconsistent with observations in UFG materials [9,34]. Microvoids and vacancy clusters have been observed by TEM inside the grain interiors of UFG $\mathrm{Cu}$ produced by HPT [9] and the UFG Al deformed by room-temperature ECAP followed by liquid nitrogen rolling [34]. Č́́žek et al. [9] have improved the DTM by considering the spatial distribution of vacancy-type defects in UFG $\mathrm{Cu}$. They assumed that after thermalization positrons enter the material uniformly. Due to the high dislocation density and proximity to the grain boundary, all positrons inside the DR were assumed to annihilate in the sinks. Since the grain diameter is comparable to the positron diffusion length, a fraction of positrons annihilate in the NR in a free or trapped state, while the rest of positrons inside the NR will diffuse from the NR to the DR.

In this study we followed the framework of the DTMs developed by Dupasquier et al. [33] and Č́ížek et al. [9]. The UFG material is assumed to be formed by identical spherical domains of radius $R$, which are divided into two regions: the boundary region with a spherical shell shape (DR) and the interior region with a spherical core shape (NR). The thickness of the DR is defined as $\delta$, which gives the radius of the NR as $R-\delta$. In addition, we make two assumptions different to the previous DTMs:

(1) Čížek et al. observed microvoids and assumed that microvoids were situated in the NR. Our positron lifetime analysis did not indicate large size vacancytype clusters. Vacancies associated with dislocations and bulk monovacancies are the major vacancy-type defects in our samples and bulk divacancies also appear in some samples.

(2) Dupasquier et al. [34] and Č́žzek et al. [9] assumed that vacancies associated with dislocations are located only in the DR due to high dislocation density near the grain boundaries in the fine-grained and UFG materials. However, we note that the concentration of vacancy-type defects depends not only on the dislocation density but also on the history of the dislocation-dislocation interactions. The macro- plastic deformation requires dislocations passing through the whole grain, which leaves a nonuniform distribution of vacancy-type defects over the grain. The current study assumes that vacancy-type defects exist in both the DR and NR with identical fractions $\left(f_{1}, f_{2}\right.$ and $\left.f_{3}\right)$. However, the defects concentrations are different in the DR and NR. The concentrations of vacancy-type defects are defined as $C_{D}$ and $C_{N}$ in the DR and NR, respectively.

There are three rate equations governing the diffusion and annihilation of positrons in the UFG samples:

First, the density of free positrons $(n)$ in the NR is given by:

$\frac{\partial n}{\partial t}=D_{+}\left(\frac{\partial^{2} n}{\partial r^{2}}+\frac{2}{r} \frac{\partial n}{\partial r}\right)-\left(\lambda_{B}+K_{N}\right) n$

where $t$ is time, $r$ is the radial distance from the center of the domain, $D_{+}$is the positron diffusion coefficient, $\lambda_{B}$ is the bulk annihilation rate for a free positron and $K_{N}$ is the positron trapping rate in vacancy-type defects in the NR.

Second, the density of positrons $\left(n_{N}\right)$ trapped in the defects situated in the NR is expressed by:

$\frac{\partial n_{N}}{\partial t}=-\lambda_{\text {trap }} n_{N}+K_{N} n_{a}$

where $\lambda_{\text {trap }}$ is the annihilation rate for trapped positrons and $n_{a}$ is the average free positron density in the NR, which can be determined by:

$n_{a}(t)=\frac{3 \int_{0}^{R-\delta} n(r, t) r^{2} d r}{(R-\delta)^{3}}$

Third, the density of positrons $\left(n_{D}\right)$ trapped in the defects situated in the DR is given by:

$\frac{\partial n_{D}}{\partial t}=-\lambda_{\text {trap }} n_{D}+\left.K_{D} n\right|_{r=R-\delta}$

where $K_{D}$ is the positron trapping rate of the vacancy-type defects in the DR.

The initial condition for Eq. (1) is:

$n(r, 0)=\frac{3(1-\eta)}{4 \pi(R-\delta)^{3}}$

where $\eta$ is the volume fraction of the DR, which can be calculated from:

$\eta=\frac{R^{3}-(R-\delta)^{3}}{R^{3}}$

It has been assumed that all positrons diffusing through the interface between the NR and the DR will annihilate in the defects in the DR. This yields a boundary condition at $r=R-\delta[9]$ :

$\left.K_{D} 4 \pi(R-\delta)^{2} \delta n\right|_{r=R}=-4 \pi(R-\delta)^{2} D_{+}\left(\frac{\partial n}{\partial r}\right)_{r=(R-\delta)}$ 
where

$\left(\frac{\partial n}{\partial r}\right)_{r=(R-\delta)}=-\frac{\alpha}{(R-\delta)} n_{r=(R-\delta)}$

and

$\alpha=\frac{K_{D} \delta(R-\delta)}{D_{+}}$

Solving Eqs. (1), (2), and (4) with the initial condition (Eq. (5)) and boundary condition (Eq. (8)) yields the following expression for the total number of positrons which annihilate in both the NR and the DR [9]:

$$
\begin{aligned}
N(t)= & 3(1-\eta) \alpha \frac{D_{+}}{(R-\delta)^{2}} \sum_{k=1}^{\infty} a_{k} \frac{\lambda_{B}-\lambda_{\text {trap }}}{\left(\lambda_{k}-\lambda_{B}-K_{N}\right)\left(\lambda_{k}-\lambda_{\text {trap }}\right)} e^{-\lambda_{k} t} \\
& +\left(\eta+3(1-\eta) \alpha \frac{D_{+}}{(R-\delta)^{2}} \sum_{k=1}^{\infty} a_{k} \frac{\lambda_{k}-\lambda_{B}}{\left(\lambda_{k}-\lambda_{B}-K_{N}\right)\left(\lambda_{k}-\lambda_{\text {trap }}\right)}\right) e^{-\lambda_{\text {trap }} t}
\end{aligned}
$$

where $\beta_{k}$ is the $k$ th solution of:

$$
\beta_{k} \cot \beta_{k}+\alpha-1=0
$$

$a_{k}=\frac{2 \alpha}{\beta_{k}^{2}+\alpha(\alpha-1)}$

$\lambda_{k}=\lambda_{B}+K_{N}+\frac{\beta_{k}^{2} D_{+}}{(R-\delta)^{2}}$

It can be seen that the first term of $N(t)$ consists of infinite exponential components, which cannot be resolved in spectra in any practical experimental situation [33,9]. If a weighted average is used to represent the first component, Eq. (10) can be approximated by [9]:

$N(t)=\left(1-I_{2}\right) e^{-\lambda_{1} t}+I_{2} e^{-\lambda_{2} t}$

with

$$
\begin{aligned}
\lambda_{1}= & \frac{1}{\tau_{1}}=\frac{\sum_{k=1}^{\infty} i_{k}}{\sum_{k=1}^{\infty} i_{k} t_{k}} \\
\lambda_{2}= & \frac{1}{\tau_{2}}=\lambda_{\text {trap }} \\
I_{2}= & \eta+3(1-\eta) \alpha \frac{D_{+}}{(R-\delta)^{2}} \sum_{k=1}^{\infty} a_{k} \\
& \times \frac{\lambda_{k}-\lambda_{B}}{\left(\lambda_{k}-\lambda_{B}-K_{N}\right)\left(\lambda_{k}-\lambda_{\text {trap }}\right)}
\end{aligned}
$$

where

$$
\begin{aligned}
& t_{k}=\left(\lambda_{B}+K_{N}+\frac{\beta_{k}^{2} D_{+}}{(R-\delta)^{2}}\right)^{-1} \\
& i_{k}=3(1-\eta) \alpha \frac{D_{+}}{(R-\delta)^{2}} a_{k} \frac{\lambda_{B}-\lambda_{\text {trap }}}{\left(\lambda_{k}-\lambda_{B}-K_{N}\right)\left(\lambda_{k}-\lambda_{\text {trap }}\right)}
\end{aligned}
$$

Dupasquier et al. [33] suggested that in practice the truncation at $k>\alpha$ in Eqs. (15) and (17) gives an acceptable result. We use a truncation of $k>2 \alpha$ to achieve a higher accuracy. The domain used in the model need not necessarily be a grain. Since the domain is related to the dislocation density [9], it should be the dislocation cell if the dislocation cells exist in the sample. TEM observations show that the dislocation cells are the major microstructural characteristics in samples RT-1 and CT-1 and they have evolved into grains after 4 ECAP passes. The mean linear size of the dislocation cells in these samples is similar to that of the grains in other samples. Both of them are of the order of $d=600 \mathrm{~nm}$. Therefore, a constant $R$ is set to $338 \mathrm{~nm}$ for all the samples by $R=d / \sqrt{\pi}$. The positron diffusion coefficient $D_{+}$is a property of the bulk material. It has been set to $123 \mathrm{~nm}^{2} \mathrm{ps}^{-1}$ [5]. Eqs. (17)-(19) predict the short positron lifetime $\left(\tau_{1}\right)$, long positron lifetime $\left(\tau_{2}\right)$ and intensity for the long positron lifetime $\left(I_{2}\right)$, respectively. There are three unknown parameters in these equations: $K_{N}, K_{D}$ and $\delta$, which are determined by matching the predicted $\tau_{1}, \tau_{2}$ and $I_{2}$ values with the experimental results. The following relative uncertainty has been used to determine the calculation accuracy:

uncertainty $=\left|\frac{\tau_{1}^{p}-\tau_{1}^{m}}{\tau_{1}^{m}}\right|+\left|\frac{\tau_{2}^{p}-\tau_{2}^{m}}{\tau_{2}^{m}}\right|+\left|\frac{I_{2}^{p}-I_{2}^{m}}{I_{2}^{m}}\right|$

where the superscript $p$ represents the predicted values and the superscript $m$ represents the values obtained from the positron lifetime analysis as listed in Table 1.

The calculated $K_{N}, K_{D}, \delta$ values and calculation uncertainties are listed in Table 2. Sample RT-1 has the largest uncertainty of $2.83 \%$, while the calculation errors of other samples are less than $0.25 \%$. This indicates that the calculation accuracies for all samples are acceptable. The thickness of the DR $(\delta)$ varies from sample to sample. Sample RT-1 has a small value of $\delta(10.2 \mathrm{~mm})$. As the deformation increases, $\delta$ increases to $106.8 \mathrm{~mm}$ in the sample RT-4 and then decreases to $52.9 \mathrm{~mm}$ in the sample RT-8. For the CTECAPed samples, CT-1 has a large $\delta$ value, while the $\delta$ values of samples CT-4 and CT- 8 are close to those of their room-temperature counterparts.

The positron trapping rate $K$ is proportional to the defect concentration $C$ :

$K=\mu C$

where $\mu$ is the specific trapping rate, which is expressed by:

$\mu \cong \sigma v$

where

$v=\sqrt{\frac{8 k T}{\pi m}}$

Table 2

Calculated $K_{N}, K_{D}, \delta$ and calculation uncertainty.

\begin{tabular}{lllrl}
\hline Sample & $K_{N}\left(\times 10^{10} \mathrm{~s}^{-1}\right)$ & $K_{D}\left(\times 10^{10} \mathrm{~s}^{-1}\right)$ & $\delta(\mathrm{nm})$ & Uncertainty $(\%)$ \\
\hline RT-1 & 1.101 & 0.580 & 10.2 & 2.83 \\
RT-4 & 0.419 & 0.988 & 106.8 & 0.138 \\
RT-8 & 4.337 & 5.389 & 52.9 & 0.035 \\
CT-1 & 1.157 & 0.790 & 110.98 & 0.0526 \\
CT-4 & 0.367 & 0.246 & 111.03 & 0.242 \\
CT-8 & 1.889 & 3.284 & 54.26 & 0.216 \\
\hline
\end{tabular}


is the average diffusion speed of the positron, $\sigma$ is the trapping cross section and $m$ is the mass of the positron [35].

At $T=300 \mathrm{~K} v$ is $1.076 \mathrm{~m} \mathrm{~s}^{-1}$. It has been assumed that a monovacancy and a divacancy possess the same volumes as the single atom and double atoms in the perfect material, respectively. The trapping cross-sections for a monovacancy and a divacancy can be calculated by the same method used in Ref. [35] using the Al lattice constant of $4.05 \AA$. The values are determined to be $0.0787 \mathrm{~nm}^{2}$ for a monovacancy and $0.1249 \mathrm{~nm}^{2}$ for a divacancy. Vacancies associated with dislocations are assumed to have the same trapping crosssection as bulk monovacancies. Therefore, the specific trapping rate is $\mu_{1 v}=8.342 \times 10^{-9} \mathrm{~cm}^{3} \mathrm{~s}^{-1}$ or $5.097 \times 10^{14} \mathrm{~s}^{-1}$ atom $^{-1}$ for bulk monovacancies and vacancies associated with dislocations, and $\mu_{2 v}=1.388 \times 10^{-8} \mathrm{~cm}^{3} \mathrm{~s}^{-1}$ or $8.481 \times 10^{14} \mathrm{~s}^{-1}$ atom $^{-1}$ for bulk divacancies. The specific trapping rate obtained for monovacancies is comparable to the value $\left(4 \pm 1 \times 10^{14} \mathrm{~s}^{-1}\right.$ atom $\left.{ }^{-1}\right)$ determined from the formation entropy [25]. Considering the multiple components of the vacancy-type defects in the NR and DR, Eq. (21) can be rewritten as:

$K_{N}=\left(f_{1} \mu_{1 v}+f_{2} \mu_{1 v}+f_{3} \mu_{1 v}\right) C_{N}$

$K_{D}=\left(f_{1} \mu_{1 v}+f_{2} \mu_{1 v}+f_{3} \mu_{1 v}\right) C_{D}$

where $C_{N}$ and $C_{D}$ are the concentrations of vacancy-type defects in the NR and DR, respectively.

The calculated $C_{N} \mathrm{~s}$ and $C_{D} \mathrm{~s}$ for the RT- and CTECAPed samples are shown in Fig. 5a and b, respectively. For the sample RT-1, $C_{D}$ is lower than $C_{N}$. Vacancies associated with dislocations are the dominant vacancy-type defects in this sample and they can easily annihilate in the jogs on the dislocations. The inter-jog distance can be estimated by $\left(\frac{v_{j}}{v \rho}\right)^{1 / 2}[36]$, where $\rho$ is the dislocation density, $v_{j}$ is the lateral jog velocity and $v$ is the mean dislocation velocity. The high dislocation density near the grain boundaries gives rise to a short inter-jog distance, leading to a smaller $C_{D}$ in the DR. In addition, vacancies near the grain boundaries annihilate easily to the latter, which is also responsible for the lower $C_{D}$ in the DR and the small width of the DR $(\delta)$ as indicated in Table 2. In the sample RT-4, $C_{D}$ is larger than $C_{N}$, indicating that bulk vacancies tend to migrate to the grain boundaries due to the low dislocation density in the NR and barriers may exist to prevent vacancies from annihilating into grain boundaries. The possible barriers sealing grain boundaries may be high-energy and nonequilibrium configurations [37] along the grain boundaries or/and nanosized precipitates in the grain boundaries [30]. The continuous migration of vacancies and the grain boundary sealing effect result in an enlarged $\delta$ in the sample RT-4. After 8 RT-ECAP passes, the defect concentrations in both the NR and DR have been significantly increased. The vacancy concentration in the DR exceeds $1 \times 10^{-4}$, which is comparable to the vacancy concentration at melting temperatures for most metals [38]. This can be contributed to the significantly strengthened grain boundary sealing effect caused by the
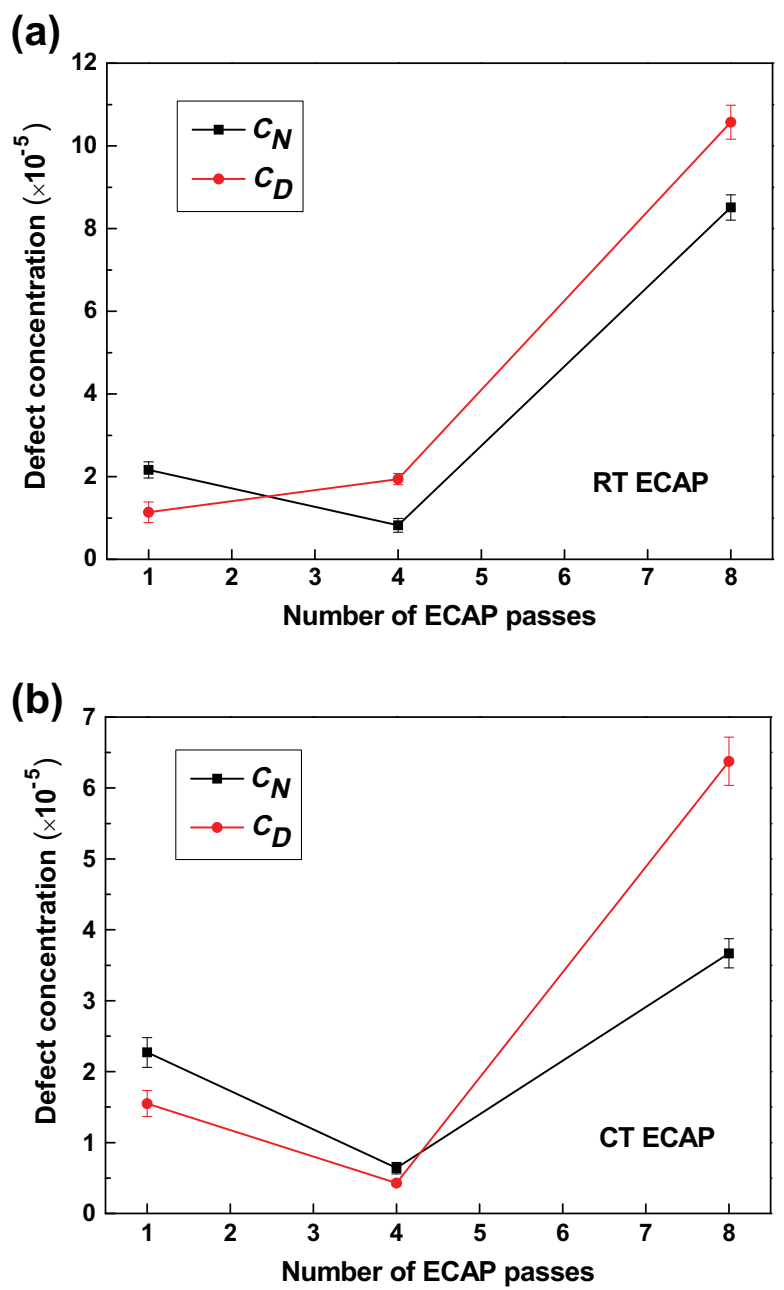

Fig. 5. Calculated $C_{N}$ and $C_{D}$ for (a) RT-ECAPed and (b) CT-ECAPed samples.

heavy deformation. Moreover, the reduced dislocation density in the grain interior results in less vacancy migration. This means that vacancies tend to concentrate in the vicinity of the grain boundaries, leading to a reduced $\delta$ in the sample RT-8 compared to the sample RT-4. The high vacancy concentration in the sample RT-8 confirms the conclusion of Ref. [3], which also identified a high vacancy concentration in $\mathrm{Al}$ deformed by more than 6 RT-ECAP passes. In this case differential scanning calorimetry and XRD were employed.

Fig. $5 b$ shows that the sample CT-1 has slightly higher vacancy concentrations than RT-1. It should be kept in mind that the dominant defects in the sample CT-1 are bulk monovacancies rather than vacancies associated with dislocations. The higher concentration of bulk monovacancies in the grain interiors of the sample CT-1 introduces a higher driving force for vacancy annihilation to grain boundaries, leading to a larger $\delta$ in the sample CT-1 than the sample RT-1. After 4 ECAP passes at cryogenic temperatures, the vacancy concentrations in both the DR and NR have decreased; this is due to the formation of divacancies. Since two monovacancies form one divacancy, the generation of 
divacancies reduces the total number of defects. In addition, bulk divacancies are more mobile than bulk monovacancies and some bulk divacancies will annihilate at grain boundaries. After 8 cryogenic passes, due to the same reasons as for the sample RT- 8 , barriers have been generated that seal the grain boundaries, resulting in supersaturated vacancytype defects. Sample CT-8 possesses lower vacancy concentrations than the sample RT-8; this may be due to the existence of divacancies in the sample CT-8. Reasons responsible for the change of the $\delta$ values in samples CT-4 and CT- 8 should be the same as samples CT-4 and CT-8, respectively, since the two groups of samples have similar $\delta$ values after 4 ECAP passes.

It can be seen in Fig. 1 that the CT-ECAPed samples are harder than the RT-ECAPed samples. However, no significant difference in microstructure has been observed between two groups of samples by TEM. It is well known that dislocation pinning by vacancy-type defects can cause material hardening. Birnbaum stated that the yield stress is related to vacancy-type defects which retained their identity on arrival at the dislocation but not the defects which have become incorporated into the dislocation structure [13]. This indicates that bulk mono- and divacancies, rather than vacancies associated with dislocations, are the effective dislocation pinning centers. Due to the interaction between effective pinning vacancies and dislocations, the material strength or hardness $(H)$ is proportional to the concentration of the effective pinning vacancy-type defects and $D^{2 / 3}$ [13], where $D$ is the diffusion coefficient of the effective vacancy-type defect. We defined a parameter $\xi$ to consider the combined influence of the defect concentration and diffusion coefficients of the effective pinning vacancies on the hardness:

$\xi=f_{2} \bar{C} D_{1 v}^{2 / 3}+f_{3} \bar{C} D_{2 v}^{2 / 3}$

$\bar{C}=\eta C_{D}+(1-\eta) C_{N}$

where $\bar{C}$ is the overall concentration of vacancy-type defects. The subscripts $1 v$ and $2 v$ represent bulk mono- and divacancies, respectively. $D$ can be expressed as [37]:

$D=g a^{2} v_{o} \exp \left(\frac{S_{M}}{k}\right) \exp \left(-\frac{E_{M}}{k T}\right)$

$v_{o}=\frac{1}{a}\left(\frac{E_{M}}{m_{a}}\right)^{1 / 2}$

where $g$ is a geometrical factor, $a$ is the lattice constant, $m_{a}$ is the mass of the atom, $v_{o}$ is the attempt frequency associated with a jump of an adjacent atom into the vacancy, $S_{M}$ is the entropy of migration of vacancy-type defects and $E_{M}$ is the activation energy of migration of vacancy-type defect. In Al, $g$ is 1 for a monovacancy and 1/6 for a divacancy, $S_{M}$ is set to $0.8 k$ and $1.2 k$ for mono- and divacancies, respectively [37], where $k$ is the Boltzmann constant. $E_{M}$ for a monovacancy is in the range of $0.61-0.64 \mathrm{eV}$ $[29,30]$, while $E_{M}$ for a divacancy is in the range of $0.42-$ $0.5 \mathrm{eV}$ [30]. In this work we have used $E_{M}=0.62 \mathrm{eV}$ for a monovacancy and $E_{M}=0.46 \mathrm{eV}$ for a divacancy.
Based on the definition of the parameter $\xi$, an equation has been developed to describe the influence of vacancytype defects on hardness:

$H=H_{0}(1+A \xi)$

where $A$ is a constant representing the dislocation pinning effect by vacancy-type defects and $H_{0}$ denotes the hardness of a vacancy-free sample. It is expected that $A$ is a constant for all samples, while $H_{0}$ depends on the dislocation density and grain size. Since TEM observations did not show significant differences in the microstructures of the RT- and CT-ECAPed samples subjected to the same number of ECAP passes, we assume that the hardness difference between these samples is due to the dislocation pinning caused by bulk mono- and divacancies. Therefore, Eq. (30) can be expressed as:

$\frac{H_{C}}{H_{R}}=\frac{1+A \xi_{C}}{1+A \xi_{R}}$

where the superscripts $C$ and $R$ denote the CT-ECAPed sample and the RT-ECAPed sample subjected to the same deformation, respectively. $H_{C}$ s and $H_{R}$ s are shown in Fig. 1 and $\xi_{C}$ s and $\xi_{R}$ s can be determined by Eq. (26) using the values listed in Tables 1 and 2. As mentioned above, bulk divacancies may exist in the sample CT-8; however, the fraction of divacancies could not be determined from the long positron lifetime. Therefore, only the constant $A$ for 1 pass and 4 pass samples has been calculated. The value $A=1.53 \times 10^{8}$ was obtained for samples RT- 1 and CT-1 and $A=1.52 \times 10^{8}$ was obtained for RT-4 and CT-4. These two results are in close agreement, confirming that bulk mono- and divacancies play a significant role in the hardening of UFG materials. If we use the average of the above $A$ values and the long positron lifetime, the fractions of three types of vacancy-type defects for the sample CT-8 can be determined using $f_{1} \times 220+f_{2} \times 245+f_{3} \times 273=$ $\tau_{2}$ and Eq. (31). This gives the values $f_{1}=22.86 \%$, $f_{2}=70.3 \%$ and $f_{3}=6.84 \%$. It confirms our assumption that bulk divacancies exist in the sample CT-8 and contribute to the materials hardening.

\section{Conclusions}

UFG Al alloys have been produced by ECAP at both room temperature and cryogenic temperature. PALS has been used to investigate vacancy-type defects in these materials and their effects on material hardening. The following conclusions can be made.

(1) The CT-ECAPed samples have higher hardness values than the RT-ECAPed samples subjected to the same deformation. The RT-ECAPed samples have equiaxed microstructure after 4 and 8 ECAP passes while the CT-ECAPed samples have slightly elongated grains and slightly smaller grain sizes. 
(2) Three types of vacancy-type defects, i.e. vacancies associated with dislocations, bulk monovacancies and bulk divacancies, have been identified in the ECAPed samples. Vacancies associated with dislocations dominate in sample RT-1, while bulk monovacancies become the dominant vacancy-type defects in samples RT-4 and RT-8. Bulk monovacancies are the major vacancy-type defects in the CT-ECAPed samples and bulk divacancies appear in the highly strained samples (CT-4 and CT-8).

(3) The increased hardness of the CT-ECAPed samples can be attributed to the existence of bulk monoand divacancies in these samples. These two types of defects are the major vacancy-type defects that can work as dislocations pinning centers and induce hardening.

(4) Positron trapping rate and vacancy-type defects concentration were calculated. A model was proposed to relate the material hardness with the concentrations of vacancy-type defects and diffusion coefficients of bulk mono- and divacancies.

\section{Acknowledgements}

L.H.S. would like to thank the China Scholarship Council (CSC) for the financial support. The positron studies were supported by the Australian Research Council Centre of Excellence Program in the Centre for Antimatter-Matter Studies. L.Z.H. would like to thank the Fundamental Research Funds for the Central Universities (N100409002), Chinese Post-doctorate Science Fund (20100471455) and State Basic Research Development Program of China (2012CB723307) for their financial support to this research.

\section{References}

[1] Argon AS, Haasen P. Acta Metall Mater 1993;41:3289.
[2] Hockauf M, Meyer LW. J Mater Sci 2010;45:4778.

[3] Su LH, Lu C, Tieu AK, He LZ, Zhang Y, Wexler D. Mater Lett 2011;65:514.

[4] Puska MJ, Nieminen RM. Rev Mod Phys 1994;66:841.

[5] Dupasquier A, Kögel G, Somoza A. Acta Mater 2004;52:4707.

[6] Č́žžek J, Procházka I, Kužel R, et al. Acta Phys Pol A 2005;107:745.

[7] Č́žžek J, Procházka I, Melikhova O, et al. Appl Surf Sci 2002;194:140.

[8] Č́̌žek J, Procházka I, Smola B, et al. Mater Sci Eng A 2007;462:121.

[9] Č́žzek J, Procházka I, Cieslar M, et al. Phys Rev B 2002;65:094106.

[10] Č́žzek J, Janeček M, Srba O, et al. Acta Mater 2011;59:2322.

[11] Divinski SV, Ribbe J, Baither D, et al. Acta Mater 2009;57:5706.

[12] Lechner W, Puff W, Mingler B, et al. Scripta Mater 2009;61:383.

[13] Birnbaum HK. J Appl Phys 1963;14:2175.

[14] Wang YM, Chen MW, Zhou FH, et al. Nature 2002;419:912.

[15] Wang YM, Ma E. Acta Mater 2004;52:1699.

[16] Zhu YT, Liao XZ. Nat Mater 2004;3:351.

[17] Chen YB, Li YL, He LZ, et al. Mater Lett 2008;62:2821.

[18] Shukla A, Hoffmann L, Manuel AA, et al. Mater Sci Forum 1997;255-257:233.

[19] Shukla A, Peter M, Hoffmann L. Nucl Instrum Meth A 1993;335:310.

[20] Hidalgo C, González-Doncel G, Linderoth S. Phys Rev B 1992;45:7017.

[21] Staab TEM, Somieski B, Krause-Rehberg R. Nucl Instrum Meth A 1996;381:141.

[22] McGuire S, Keeble DJ. J Phys D 2006;39:3388.

[23] Petersen K, Repin IA, Trumpy G. J Phys: Condens Matter 1996;8:2815.

[24] Rajainmäkit H, Linderoth S. J Phys: Condens Matter 1990;2:6623.

[25] Fluss MJ, Smedskjaer LC, Chason MK, et al. Phys Rev B 1978; $17: 3444$

[26] Häkkinen H, Mákinen S, Manninen M. Phys Rev B 1990;41:12441.

[27] Puska MJ, Nieminent RM. J Phys F: Met Phys 1983;13:333.

[28] Damask AC, Dienes GJ. Point defects in metals. New York: Gordon \& Breach; 1971.

[29] Mishin Y, Farkas D, Mehl MJ, et al. Phys Rev B 1999;59:3393.

[30] Saimoto S, Cooley J, Larsen H, et al. Philos Mag 2009;89:853.

[31] Chang JY, Kim GH, Moon IG. Scripta Mater 2001;44:331.

[32] Brandt W, Paulin R. Phys Rev B 1972;5:2430.

[33] Dupasquier A, Romero R, Somoza A. Phys Rev B 1993;48:9235.

[34] Wu XL, Li B, Ma E. Appl Phys Lett 2007;91:141908.

[35] Petersen K, Thrane N, Trumpy G. Appl Phys 1976;10:85.

[36] Nicholas JF. Acta Metall 1959;7:544.

[37] Horita Z, Smith DJ, Nemoto M, et al. J Mater Res 1998;13:446.

[38] Seeger A, Schumacher D, Schilling W, et al. Vacancies and interstitials in metals. Amsterdam: North-Holland; 1970. 